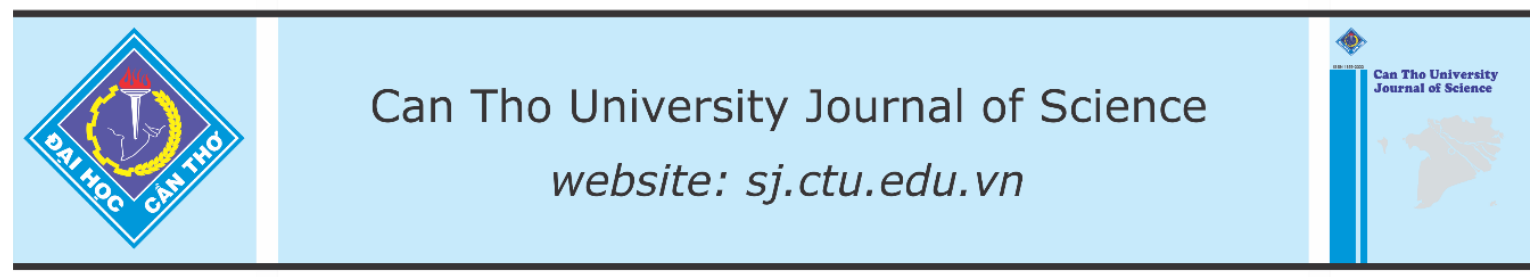

DOI: 10.22144/ctu.jen.2021.001

\title{
Controlling the external device in real-time using eeg brain signals based on eyes states
}

\author{
Nguyen Hoang Dung ${ }^{1 *}$, Nguyen Co Thach ${ }^{1}$, Huynh The Hien ${ }^{2}$ and Nguyen Mai Ngan ${ }^{1}$ \\ ${ }^{1}$ Department of Automation Technology, Can Tho University, Vietnam \\ ${ }^{2}$ Department of Control, PetroVietnam Ca Mau Fertilizer JSC, Ca Mau, Vietnam \\ *Correspondence: Nguyen Hoang Dung (email: hoangdung@ctu.edu.vn)
}

\section{Article info.}

Received 30 Aug 2020

Revised 08 Dec 2020

Accepted 31 Mar 2021

\section{Keywords}

Brain-computer interfaces, eyes states, feature extraction, Support Vector Machine classification, electroencephalography

\section{ABSTRACT}

The paper proposes a new method to control external device in real-time using electroencephalography-based brain signals. The brain signals of a healthy female student at F7 and F8 channels are recorded from Emotiv Epoc+. They are filtered using a combination of wavelet approach and recursive least square estimation to remove unwanted noises. Open and closed eyes states are extracted from filtered brain signals. The support vector machine approach is applied to classify two states of eyes (open and closed). The classified eyes states are utilized to generate the on and off commands, respectively. Those commands are sent to an Arduino control board to control on and off states of the light. Experimental results showed that the average accuracy of two control commands is $81.6 \%$. The obtained results promise for extraction of more commands that can be utilized for applications in daily life.

\section{INTRODUCTION}

Electroencephalography (EEG) can detect human brain activity from millions of neurons (Illes \& Sahakian, 2011). Electrodes are put human scalp to get the EEG signal in microvolt (Lopes da Silva, 2013). At the first time, EEG could be utilized to predict several relevant brain diseases such as epilepsy and sleeping disorders, tumors, strokes, other brain disorders (Barbara et al., 2013; William et al., 2014). After that, the birth of functional magnetic resonance imaging (fMRI), near-infrared spectroscopy (fNIRS) (Hong \& Nguyen, 2014; Nguyen \& Hong, 2014, 2015a, 2015b, 2016) and computed tomography scan (CT) are utilized to effectively detect brain disorders. EEG has low spatial resolution but high temporal resolution while fMRI is high spatial resolution but low temporal resolution (Nguyen et al., 2018).
Movement activities in daily life are really significant for human. However, some people are not able to move because of their accidents, brain-muscle disorders (Vaibhav, 2014), and amyotrophic lateral sclerosis (ALS) characterized by stiff muscles. Even though their movements are difficult, their brain still works well. A brain computer-interface (BCI) can help them to communicate with the surrounding environment. $\mathrm{BCI}$ is a neural-control interface or a communication pathway between brain and external devices. The first work on BCI was reported in 1970s (Vidal, 1973). EEG based BCI consists of recorded brain signals, preprocessing data to remove noises, specific features extraction, classification, control command generation, and feedback signals (Bashashati et al., 2007). For example, BCI was utilized to enter characters from human though without using keyboard (Townsend et al., 2010). Recently, several works have proposed that brain 
signals could be utilized to control the external device (Nguyen and Huynh, 2018) based on human thought (Ha Hoang Kha et al., 2016) or face expression (Nguyen et al., 2019).

In the current work, EEG based brain signals from eyes' states (open and closed) can be utilized to control the external devices. Firstly, EEG based brain signals are recorded from channels of F7 and F8 using Emotive Epoc+. Secondly, noises in brain signals will be eliminated by combination of wavelet approach and recursive least square estimation. Then specific features of closed and open states of eyes are extracted for classification using the algorithm of support vector machine (SVM). Finally, two commands are sent to Arduino control board to drive a light with two states (On and Off) in realtime.

\section{MATERIALS AND METHODOLOGY}

\subsection{The algorithm for controlling external device}

Figure 1 presents the algorithm scheme for controlling external devices based on EEG brain signals. EEG Emotiv Epoc with 14 channels is utilized to record brain signals that are sent to computer with the Bluetooth protocol. Noises from the recorded EEG data are removed using the RLS adaptive algorithm. The $2 \mathrm{~s}$ window frame is applied to filtered EEG data before going to extract the features of open and closed eye states. The SVM approach is utilized to classify two states of open and closed eyes. Two commands are generated from the brain EEG signals according to the eye states are sent to an arduino control board based on the serial protocol. Finally, when the arduino control board receives the commands of open and closed eye states, it turns on and off the light, respectively.

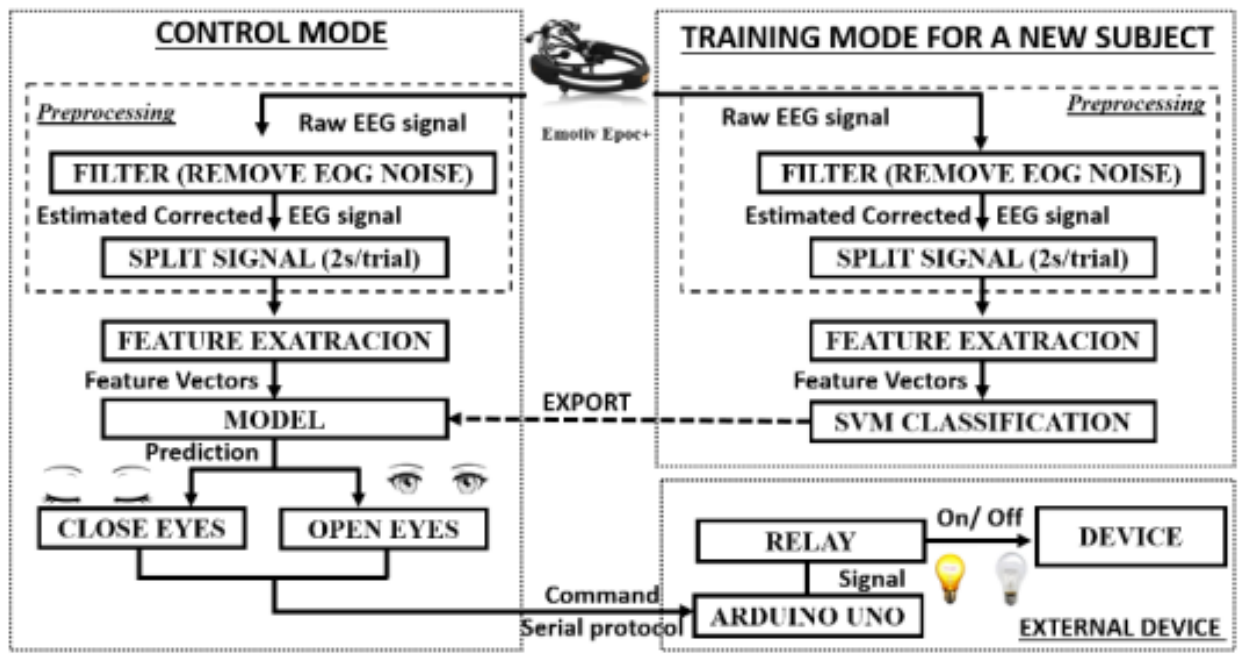

Fig. 1. The scheme for controlling external devices based on EEG brain signals

\subsection{Experimental paradigm}

A 22-year-old female student with good health and without relevant brain disorders was invited to participate in the experiment with 400 times of open and closed eyes (200 for open eyes and other 200 for closed eyes) with 2 second closed eyes followed by 2 second open eyes. Experimental paradigm was designed on computer to make a beep sound to let her know for state changes of open and closed eyes. She was clearly instructed before doing experiment. Especially, the experiment was conducted in quite room to limit the noises. Also, she was asked to relax and did not move her body during the experiment. Emotive Epoc+ with 14 channels and sampling rate of $128 \mathrm{kHz}$ (AF3, F7, F3, FC5, T7, P7, $\mathrm{O} 1, \mathrm{O} 2, \mathrm{P} 8, \mathrm{~T} 8, \mathrm{FC} 6, \mathrm{~F} 4, \mathrm{~F} 8$, and AF4) and two reference channels (P3 (CMS)/P4 (DRL)) was put on her head according to the 10-20 international system (Figure 2) such that electrodes well contacting with her scalp. The data of channels F7 and F8 (red open circles in Figure 2b) are selected because their positions are near to eyes regions. 

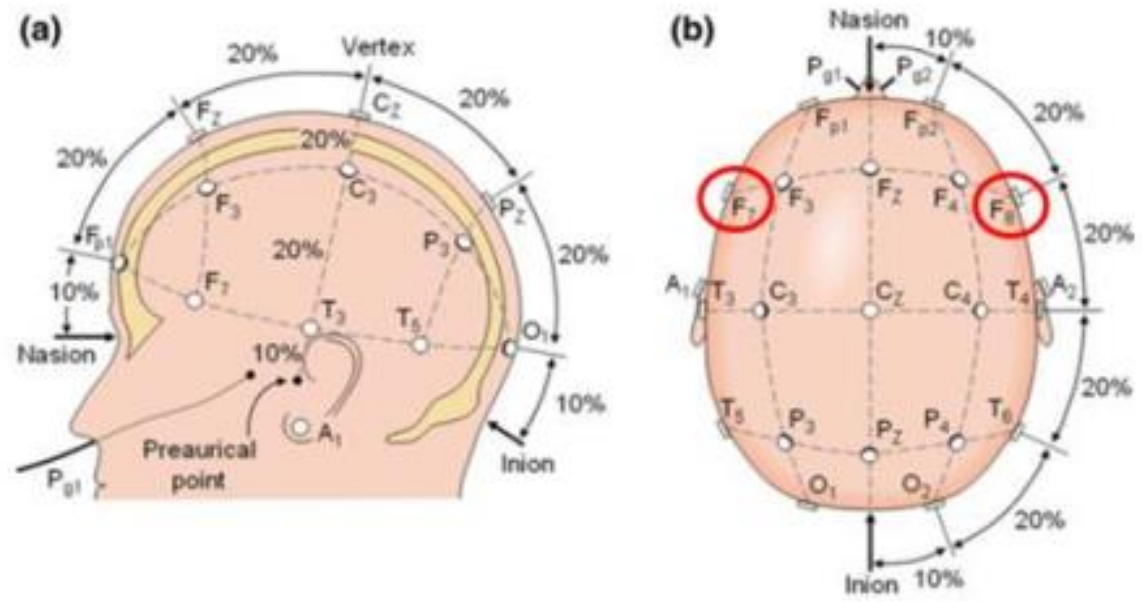

Fig. 2. The brain signals of eyes states recorded from channels of F7 and F8 according to the 10-20 international systems (Jasper, 1958)

P3 (CMS) and P4 (DRL) are two reference electrode channels, 14 other electrodes located at AF3, F7, F3, FC5, T7, P7, O1, O2, P8, T8, FC6, F4, F8, and AF4 shown in Figure 2. Voltage signals from electrodes are amplified and filtered by a high-pass filter and a Notch filter with the cut-off frequency of $50 \mathrm{~Hz}$.

\subsection{Data preprocessing}

EEG based brain signals are affected by many kinds of noises such as experimental environment, physiology noises, and noises from equipment. Therefore, the noise removal is really significant to get good brain signal. In the current work, a combination of wavelet and RLS estimation are utilized to eliminate noises in EEG based brain signals.

The RLS adaptive filter based on wavelet decomposition is utilized to eliminate noises of eyes blinking in the coarse electroencephalogram chain proposed in the previous study (Peng et al., 2013). The RLS method is believed to be fast, efficient, and suitable to apply for real-time applications.

\section{Wavelet decomposition}

EEG based brain signals are decomposed two components: Approximation (A) respects to low frequency component and Detail (D) illustrates for high frequency component according to low pass filter and high pass filter, respectively (Figure 3). Filters are conducted in different layers. To reduce computing time, the signals will be down-sampled two times if they are passed filters. Wavelet Daubechies with the $4^{\text {th }}$ orders $(\mathrm{db} 4)$ available in MATLAB is utilized to decompose the brain signals.

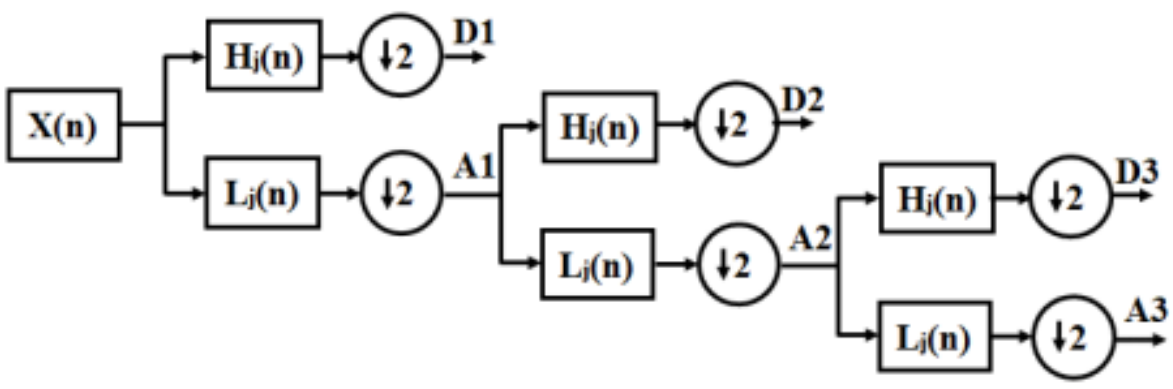

Fig. 3. Separation of EEG data using discrete wavelet transform 
Adaptive filter based on wavelet decomposition and RLS

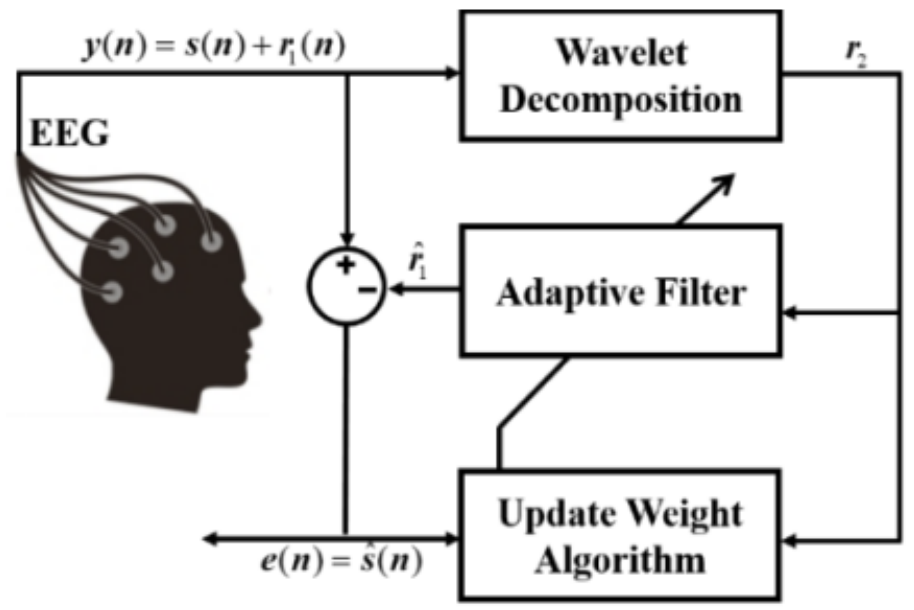

Fig. 4. Diagram of adaptive filter to eliminate blinking noise (Peng et al., 2013)

Figure 4 presents the diagram of adaptive filter to eliminate blinking noise. Firstly, the input EEG signal is decomposed using wavelet transform with a mother wavelet function $\mathrm{db} 4$ available illustrated in Figure 3. The wavelet approach is utilized to separate the seven levels from the recorded brain signal, $y$ (including the clean EEG signal $S$ and the blinking noise signal $r_{l}$ ). Secondly, the noise approximation signal EOG $r_{2}$ is utilized as a reference signal for the adaptive filter. Finally, the clean EEG signal is derived by subtracting the output of adaptive filter. The clean EEG signals are estimated using (1).

$$
e(n)=\hat{S}_{n}=y(n)-\hat{r}_{1}
$$

Where, $y(n)$ is the EEG signal obtained from the Emotiv headset, $r_{l}$ is the output of the adaptive filter. The RLS algorithm is applied for the adaptive filter reported elsewhere (Peng et al., 2013).

$$
\begin{aligned}
& \hat{r}_{1}(n)=w(n) r_{2}(n) \\
& w(n+1)=w(n)+g(n) e(n) \\
& g(n)=\frac{P I(n)}{\lambda+P I(n) P(n)} \\
& P(n+1)=\frac{P(n)-g(n) P I(n)}{\lambda}
\end{aligned}
$$

where, $P I(n)$ refers a cross-correlation matrix and $P I(n)=r_{2}(n) P(n)$. And $P 0=\delta I, \delta$ is a large enough positive coefficient, $g(n)$ denotes gain vec- tor, $w(n)$ stands for weight vector and $\lambda$ is the forgetting factor. It is usually chosen from 0.95 to 0.98 (Maddirala \& Shaik, 2016).

\subsection{Feature extraction}

Two states of open and closed eyes are extracted from filtered EEG signals. In the current work, the root mean square (RMS) of filtered EEG signal is computed for each trial. The RMS based feature-extraction method is proposed to classify the status of closed eyes and open eyes. The RMS feature values is determined as follows.

$$
F_{R M S}=\sqrt{\frac{1}{N} \sum_{n-1}^{N} s(n)^{2}}
$$

Where, $S(n)$ is $n^{\text {th }}$ signal in the EEG signal sequence and $N$ is the total discrete samples of the signal sequence.

\subsection{Classification}

Support Vector Machine (SVM) was proposed by the previous work (Bhuvaneswari \& Satheesh Kumar, 2013) to classify the problem into two or more groups. The SVM is used to construct the optimal hyperplane with largest margin for separating data between two groups. For two-dimensional data, single hyperplane is enough to separate the data in two groups such as +1 or -1 . Two hyperplanes are needed to separate the data points for three-dimensional data (Byun \& Lee, 2002).

The SVM with hyperplane structure is utilized to separate sample data based on the target categories. For two-dimensional data, there are a number of 
possible linear separators (hyperplanes) and need to find the optimal hyperplane which has maximum margin width. Suppose that a training dataset of $n$ points is given as $\left(\vec{x}_{1}, y_{1}\right), \ldots,\left(\vec{x}_{n}, y_{n}\right)$, where the $y_{\mathrm{i}}(\mathrm{i}=1, \ldots, n)$ has the value of either 1 or -1 to indicate the class which $\vec{x}_{i}$ exists. $\vec{x}_{i}$ is a real vector that the "maximum-margin hyperplane" should be definded. The nearest point $\vec{x}_{i}$ from the group is maximized (https://en.wikipedia.org/wiki/Support_vector_machine).

The SVM approach is applied in many applications in biomedical engineering such as using the EEG based cancer identification, seizure prediction, and facial and speech recognition. Actually, EEG data set is quite complex, so it is not easy for classification. However, the K kernel function can be used for separation by mapping data in a space with a greater dimension space. Some common kernel functions are shown in Table 1. In the current work, the RBF Kernel (Gaussian) function available in MATLAB is utilized to classify EEG signals because of its best classification results.
Table 1. Several popular Kernel functions

\begin{tabular}{ll}
\hline Kernels & Functions \\
\hline Linear Kernel & $K\left(x_{i}, x_{j}\right)=x_{i}^{T} x_{j}$ \\
Polynomial & $K\left(x_{i}, x_{j}\right)=\left(\begin{array}{c}{ }^{\gamma} x_{i}^{T} x_{j} \\
\text { Kernel }\end{array}\right.$ \\
$\begin{array}{l}\text { Radial basis } \\
\text { function Kernel }\end{array}$ & $K\left(x_{i}, x_{j}\right)$ \\
(RBF Kernel- $(\gamma$ & $=\exp \left({ }^{-\gamma}\left\|x_{i}-x_{j}\right\|^{2}\right)$ for $(\gamma$ \\
Gaussian) & $>0)$
\end{tabular}

\subsection{Controlled device}

The results from the SVM classification model are utilized to create the BCI system that can control the external device (Figure 5). The classification commands are sent to the Arduino control board via MATLAB software. This control unit is responsible for reading classification commands and generating control signals for external device.

- If eyes are closed, then the Light is OFF

- If eyes are opened, then the Light is ON

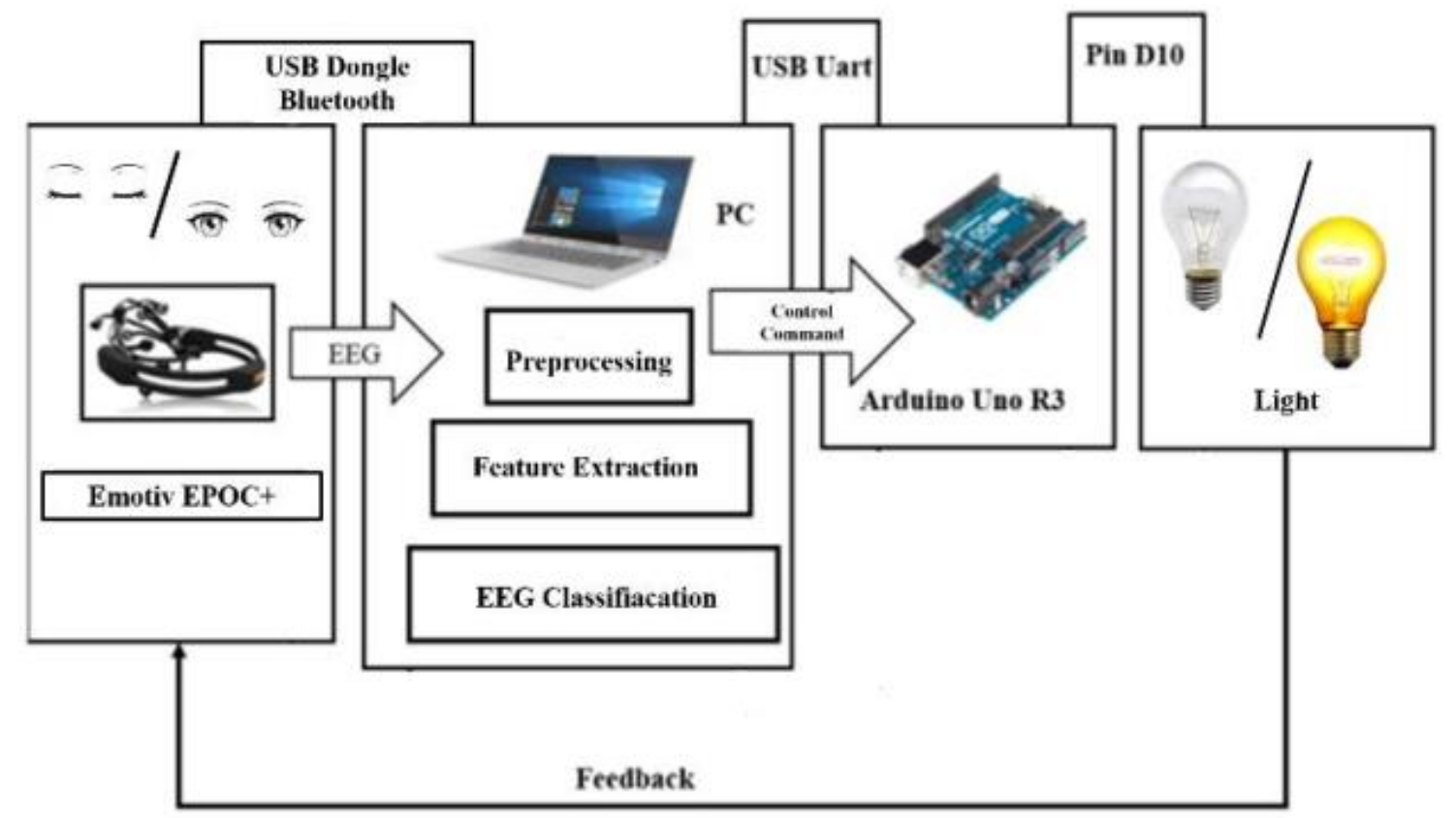

Fig. 5. Method to control the external device by EEG signal based on eye states 


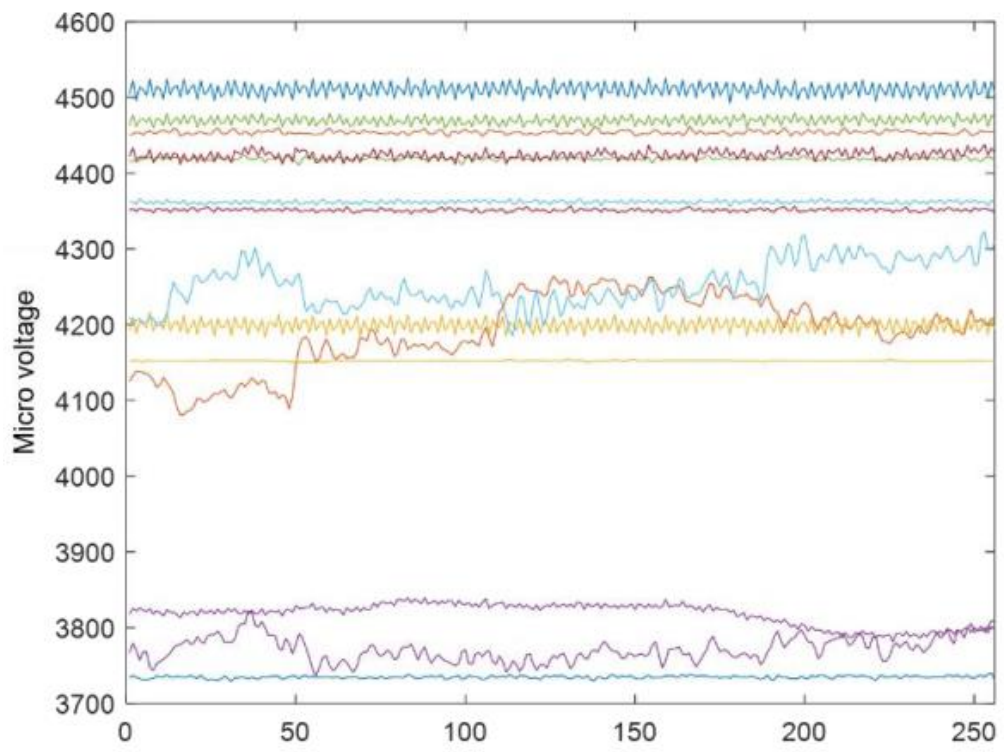

Fig. 6. EEG recording at 14 channels with open eyses states from Emotiv Epoc+ headset

\section{RESULTS AND DISCUSSION}

\subsection{EEG data}

Figure 6 presents 14 channels with the raw EEG signals recorded from the female volunteer with open eyes. This data set are recorded by Emotiv Epoc+ and without using any filters. Then noises from channels F7 and F8 are removed using adaptive filtering (combination of wavelet approach and RLS method) before applying for feature extraction and classification stages.

\subsection{Data preprocessing}

EEG data are filtered by combination of wavelet and RLS approaches to remove unwanted noises. Particularly, the EOG artifact is detected and estimated by the digital wavelet (DW) filter. The filtered one is used as a reference signal for the RLS adaptive filter to estimate noises for obtaining the original brain signal. The clean EEG is estimated for the feature extraction shown in Figure 7.
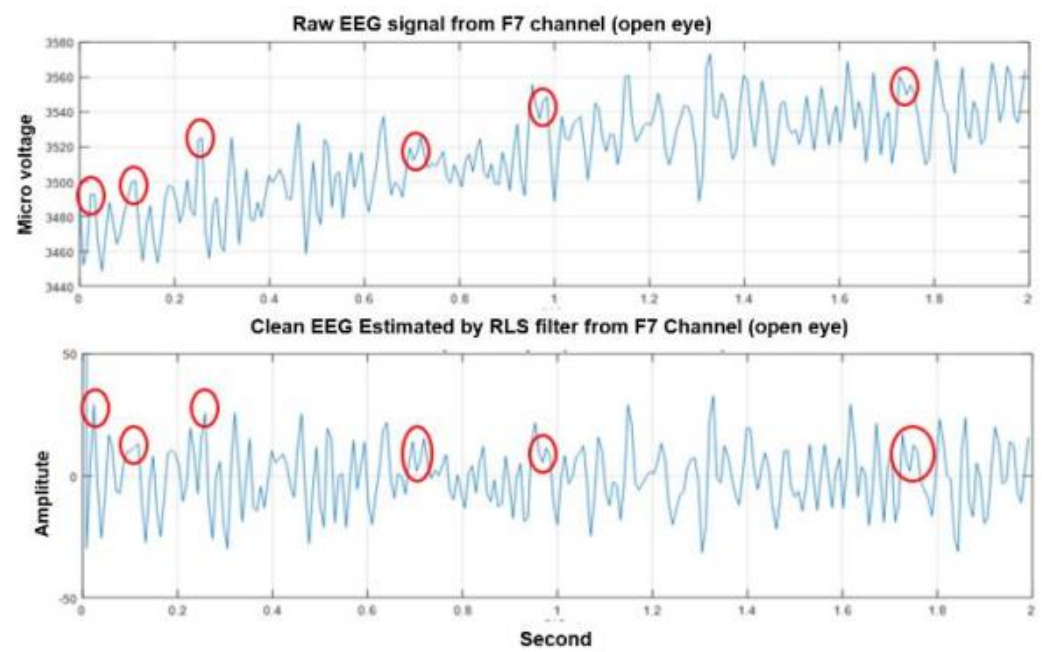

Fig. 7. Filtered EEG signal (lower panel) from the recorded EEG data of chaanel F7 using DW and RLS adaptive filter methods 


\subsection{Feature extraction}

$F_{R M S}$ from (6) is obtained by using the RMS based feature extraction technique. $F_{R M S}$ a feature vector with size $\mathrm{M} \times \mathrm{N}$ where $\mathrm{M}$ is the total number of trials samples (number of EEG data samples) is 400 , and
$\mathrm{N}$ is the total number feature values in two channels of F7 and F8. For each trial of each channel, the difference of the $F_{R M S}$ characteristic between the open or closed states has different values. Figure 8 illustrates the distribution diagram of the $F_{R M S}$ feature from two channels F7 and F8.

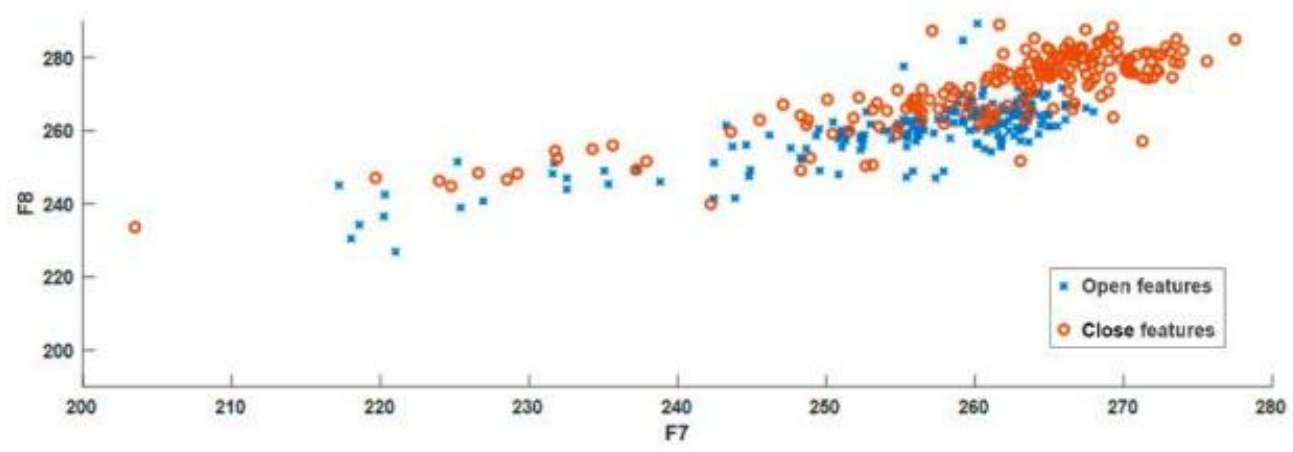

Fig. 8. FRMS feature distribution diagram of two closed/open eyes features

\subsection{Classification of open and closed eyes}

For each trial in 2 seconds, feature extraction vectors are computed. In this experiment, there are 400 feature vectors shown in Figure 9.

To assess the accuracy of the classification, $90 \%$ of the data sample is utilized for training and the remaining $10 \%$ for test. The classification methods based on approaches of Decision Tree, KNN (knearest neighbor), and SVM available in MATLAB were checked to find a classification model with the highest accuracy by using Classification LearnerScatter Plot (Figure 10). Those approaches were reported elsewhere (Tyagi, 2019) and (Yassin et al., 2020).

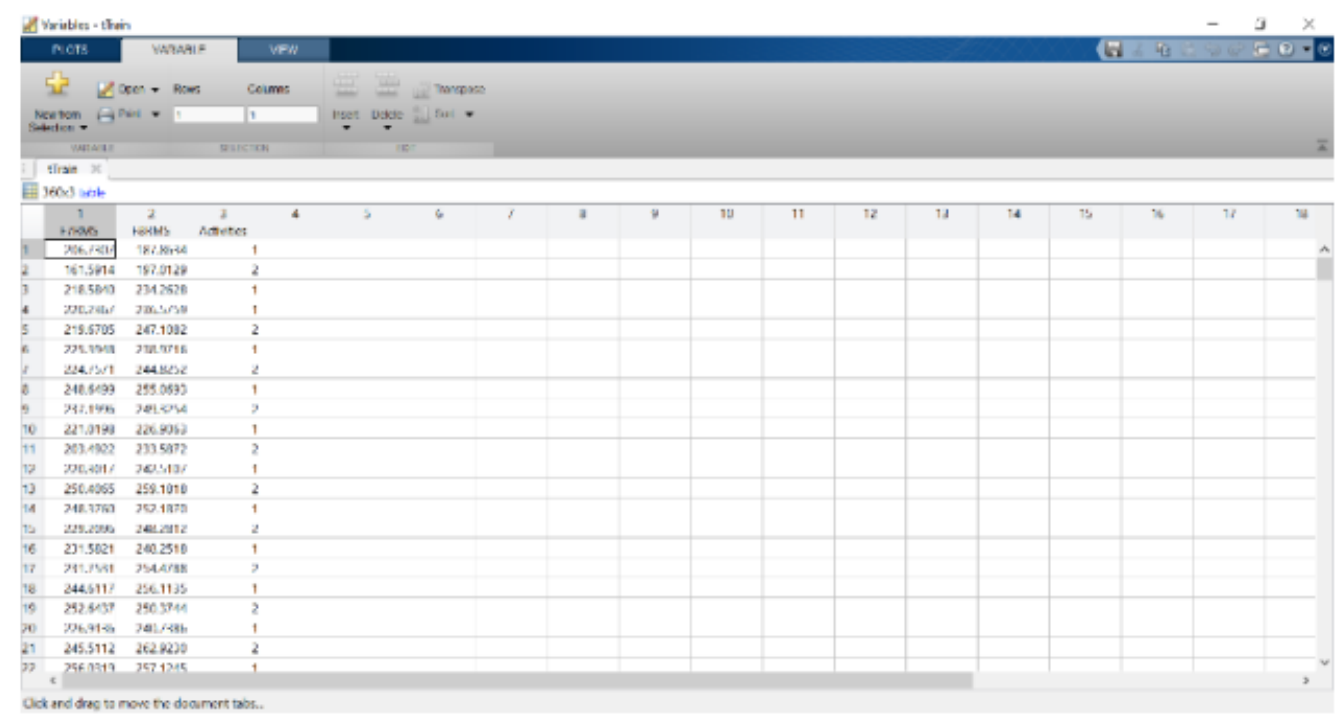

Fig. 9. Feature vectors of closed and open eyes 


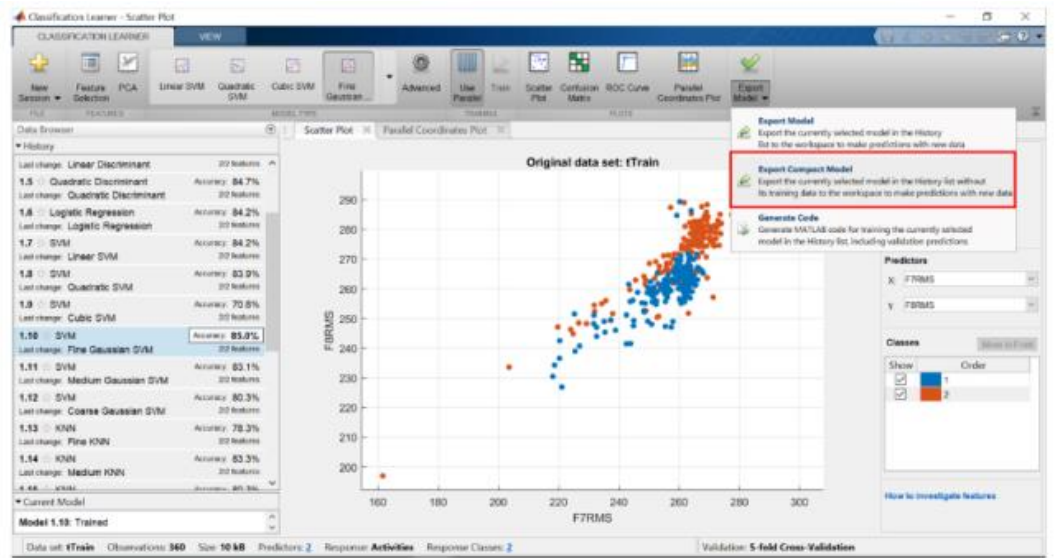

Fig. 10. Classifying two eye states based on the SVM approach (blue and red points are open and closed eye states, respectively)

The experimental results show that the online classification of two states of open and closed eyes using the the SVM algorithm with Fine Gaussian function gives the average accuracy of $81.6 \%$ (Figure 10). Esspecially, the commands of open and closed eyes from this classifiction are utilized to control the light on and off in real-time, respectively.

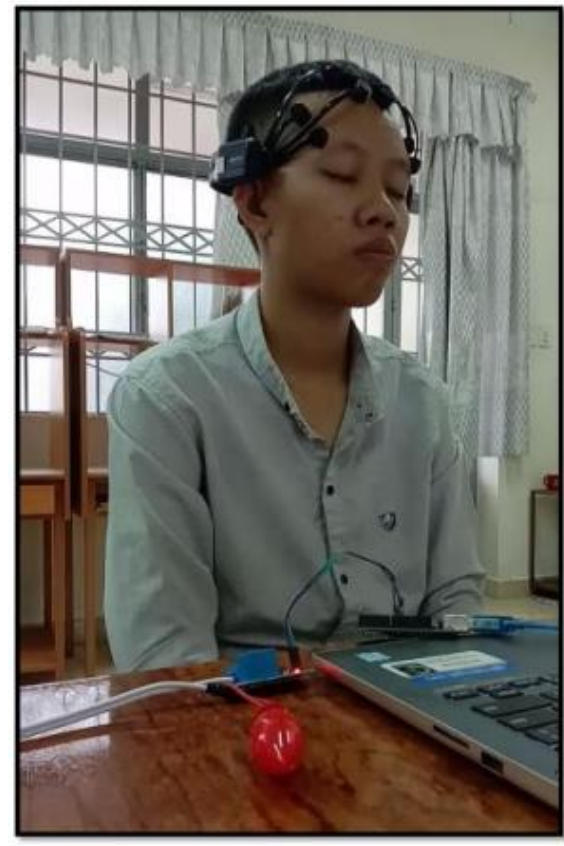

(a)

\subsection{Controlling external device based on EEG signal}

An experiment was conducted with a new volunteer male student (22-year-old). He has no history of mental disorders. At the period of the experiment, he has good cognitive ability and good health status. And the experiment was done at the intelligent system laboratory (Department of Automation Technology, Can Tho University, Vietnam) in the case of the low noise environment shown in Figure 11.

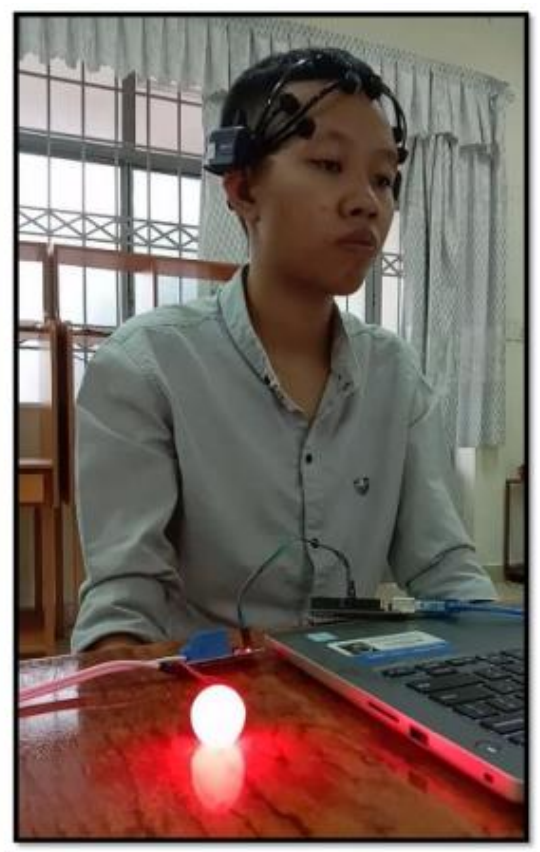

(b)

Fig. 11. Real-time control for the external device using EEG signal (a) closed eyes command, (b) open eyes command 
The experiment was conducted in two scenarios: two closed-open eyes states with cyclic and randomized period.

\section{Cyclic period scenario}

The two open-close states are implemented by user in cycles. The closed eyes state follows by the 2second open eyes state. After the 2-second, a "beep" sound is generated to inform the experimenter to change the state from open eyes to closed eyes to control the bulb light. Closed and open eyes are corresponding to the logic of level 0 and 1 , respectively. Figure 12 shows the comparison of the desired (blue solid line) and tested (red dashed line) responds of two states. And Table 2 summarizes experimental results of 30 samples for two states with the accuracy of $83.3 \%$ and $80 \%$, respectively.

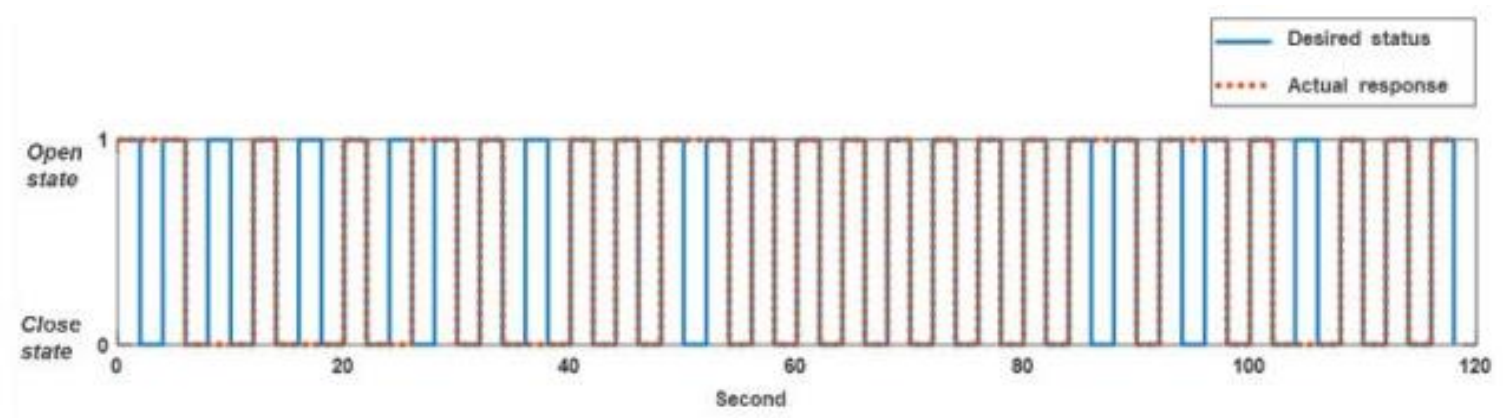

Fig. 12. Comparison of desired and actual responds with cyclic period scenario

\section{Randomized period scenario}

In this scenario, when starting a sampling period with a "beep" sound, the subject is informed to perform open his eyes. Then he was asked to close his eyes when the "beep" sound appears again. The cycle is randomly repeated with two different eyeclosed/open states. The experimental results in this scenario method are shown in Figure 13 and Table 2 with the average accuracy of $81.6 \%$.

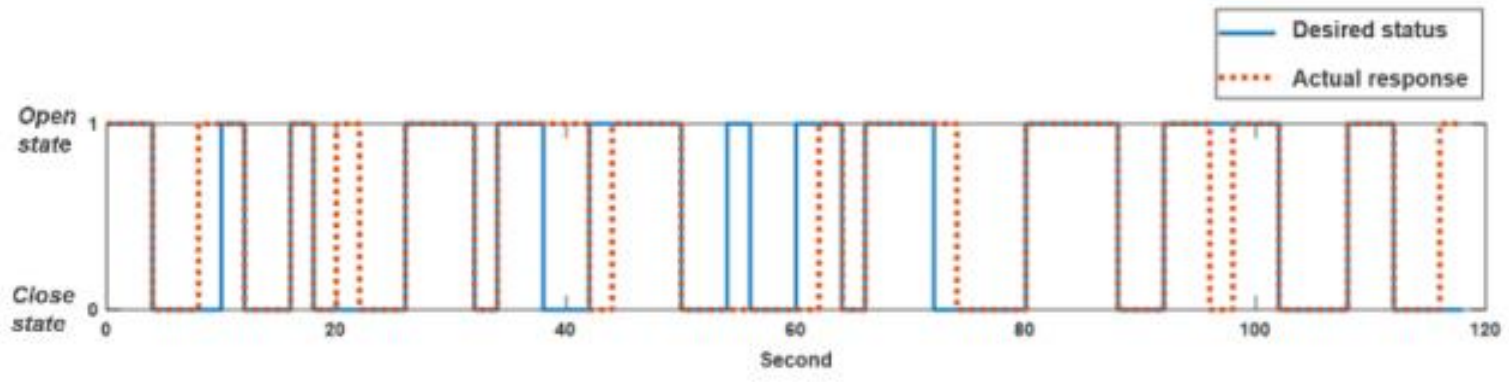

Fig. 13. Comparison of desired and actual responds with randomized period scenario

Table 2. Controlling the light based on two eyes states

\begin{tabular}{lccrr}
\hline $\begin{array}{l}\text { Cyclic period scenario } \\
\text { Commands }\end{array}$ & Samples & Correct & Wrong & Accuracy(\%) \\
\hline Open & 30 & 25 & 5 & 83.3 \\
Closed & 30 & 24 & 6 & 80.0 \\
\hline Randomized period scenario & & & 4 & 83.3 \\
\hline Open & 30 & 26 & 7 & 80.0 \\
Closed & 30 & 23 & & 81.6 \\
\hline Average accuracy & & & \\
\hline It is noted that the accuracy of open and closed eyes & It is obtained from the classification result using the \\
states listed in Table 2 is not computed from a num- & SVM approach. Therefore, 25 or 26 correct detec- \\
ber of correct identification results from 30 samples. & tion gives the same accuracy of 83.3\% (Table 2).
\end{tabular}




\section{CONCLUSIONS}

In this paper, two states of eyes recorded from EEG based brain signals were utilized to control on and off states of the light, respectively. The obtained EEG brain signals were filtered by combination of wavelet and RLS approaches. Then two states of closed and open eyes were extracted and classified using the SVM method. The experimental results showed that the average accuracy of the proposed method in both scenarios was $81.6 \%$. The proposed approach is promised for daily activities of invalids. The limitation of this work is only one subject invited to participate in the experiment because of not being easy to persuade a person allowing to measure their brain in the short time. However, in the future work, several subjects will be asked to participate to strengthen the proposed method.

\section{ACKNOWLEDGMENTS}

We would like to thank Ms. Tan Le, Mr. Meverick Nguyen, and Ms. Emy Nguyen from Emotiv (Emotiv Systems, USA) for supporting the brain recording device in our experiment.

\section{REFERENCES}

Illes, J., \& Sahakian, B. J. (Eds.). (2013). Oxford handbook of neuroethics, Oxford, UK: Oxford University Press.

Lopes da Silva, F. (2013). EEG and MEG: Relevance to Neuroscience. Neuron, 80(5), 1112-1128.

William, O.T., Aatif, M.H., Selim, R.B., and Peter W.K. (2014). Handbook of EEG interpretation. Demos Medical Publishing.

Nguyen, H.-D. and Hong, K.-S. (2016). Bundled-optode method in functional near-infrared spectroscopy. PLoS ONE, 11(10): e0165146.

Nguyen, H.-D. and Hong, K.-S. (2016). Bundled-optode implementation for 3D imaging in functional nearinfrared spectroscopy, Biomedical Optics Express, 7(9):3491-3507.

Hong, K.-S. and Nguyen, H.-D. (2014). State-space models of impulse hemodynamic responses over motor, somatosensory, and visual cortices. Biomedical Optics Express, 5(6), 1778-1798.

Nguyen, H.-D. and Hong, K.-S. (2015a). Optimizing a hemodynamic model in the human motor cortex. 2015 IEEE International Conference on Advanced Intelligent Mechatronics (AIM). July 7-11, Busan, Republic of Korea, 71-176.

Nguyen, H.-D. and Hong, K.-S. (2015b). Multiple optodes configuration for measuring the absolute hemodynamic response using spatially resolved spectroscopy method: an FNIRS study. $201515^{\text {th }}$ International Conference on Control, Automation and
Systems (ICCAS 2015). Oct, 13-16, BEXCO, Busan, Republic of Korea, 1827-1832.

Nguyen, H.-D., Yoo, S.-H., Bhutta, M.R., and Hong, K.-S. (2018). Adaptive filtering of physiological noises in fNIRS data. Biomedical engineering online, 17(1), 180.

Vaibhav Gandhi (2014). Brain-computer Interfacing for Assistive Robotics Electroencephalograms, Recurrent Quantum and User-Centric Graphical Interfaces. 1 st Editon, Academic Press; 1st Edition (October 8, 2014).

Vidal, J.J. (1973). Toward direct brain-computer communication. Annual Review of Biophysics and Bioengineering, 2, 157-180.

Bashashati, A., Fatourechi, M., Ward, R.K., and Birch G.E. (2007). A survey of signal processing algorithms in brain-computer interfaces based on electrical brain signals, Journal of Neural Engineering, 4(2), R35-57.

Townsend, G., LaPallo, B. K., Boulay, C. B., Krusienski, D. J., Frye, G. E., Hauser, C., ... \& Sellers, E. W. (2010). A novel P300-based brain-computer interface stimulus presentation paradigm: moving beyond rows and columns. Clinical neurophysiology, 121(7), 1109-1120.

Nguyen, H.-D. and Huynh, T.H. (2018). Controlling the Position of the Carriage in Real-Time Using the RBF Neural Network Based PID Controller. The $18^{\text {th }}$ International Conference on Control, Automation and Systems (ICCAS 2018). Oct. 17 20; YongPyong Resort, PyeongChang, GangWon, Korea, 1418-1423.

Kha, H.H., Kha, V.A., and Hung, D.Q. (2016). Brainwave-Controlled Applications with the Emotiv EPOC Using Support Vector Machine. 2016 3rd International Conference on Information Technology, Computer, and Electrical Engineering (ICITACEE), Semarang, Indonesia, 19-20 Oct.

Nguyen, H.-D., Nguyen, M.N., and Huynh, T.H. (2019). "Controlling Robot Arm by Brain Waves Based on Facial Expressions," Proceeding of $15^{\text {th }}$ International Conference on Multimedia InformationTechnology and Applications (MITA2019) ISSN: 1975-4736, 515-520.

Jasper, H. (1958). The ten-twenty electrode system of the International Federation. Electroencephalography and Clinical Neurophysiology, 10: 371-375.

Peng, H., Hu, B., Shi, Q., Ratcliffe, M., Zhao, Q., Qi, Y., \& Gao, G. (2013). Removal of ocular artifacts in EEG-An improved approach combining DWT and ANC for portable applications. IEEE journal of biomedical and health informatics, 17(3), 600-607.

Maddirala, A.K. and Shaik, R.A. (2016). Removal of EOG Artifacts From Single Channel EEG Signals Using Combined Singular Spectrum Analysis and Adaptive Noise Canceler. IEEE Sensors Journal, 16(23), 8279-8287. 
Bhuvaneswari P. and Satheesh Kumar J. 2013. Support Vector Machine Technique for EEG Signals. International Journal of Computer Applications, 63(13), 0975-0980.

Byun, H. and Lee, S.W. (2002). Applications of Support Vector Machines for Pattern Recognition: A Survey. In: Lee SW., Verri A. (eds) Pattern Recognition with Support Vector Machines (SVM). Lecture Notes in Computer Science, vol. 2388. Springer, Berlin, Heidelberg. https://doi.org/10.1007/3-540-45665-1_17.
Vaishali Tyagi (2019). A Review on Image Classification Techniques to classify Neurological Disorders of brain MRI. 2019 International Conference on Issues and Challenges in Intelligent Computing Techniques (ICICT). 27-28 Sept. 2019, GHAZIABAD, India.

Walid Yassin, Hironori Nakatani, Yinghan Zhu, et al. (2020). Machine-learning classification using neuroimaging data in schizophrenia, autism, ultra-high risk and first-episode psychosis. Translational Psychiatry, 10: AN. 278. 